\title{
Optical Rotation of Linearly Polarized Light Propagating through a Nonideal 1D-Superlattice
}

\author{
Vladimir V. Rumyantsev, Stanislav A. Fedorov \\ Galkin Donetsk Institute for Physics and Engineering of National Academy of Sciences, Donetsk, Ukraine. \\ Email: rumyants@teor.fti.ac.donetsk.ua \\ Received January $13^{\text {th }}, 2010$; revised January $28^{\text {th }}, 2010$; accepted February $1^{\text {st }}, 2010$.
}

\begin{abstract}
The problem of finding polariton modes (necessary for calculating gyrotropic characteristics) in space-dispersed superlattices is not yet solved. At the same time the specified quantities can be approximately evaluated if the widths of layers comprising a multilayer material are much bigger then the characteristic scales of space dispersion. In such a case the contribution of individual layers to gyrotropy can be regarded as independed. Thus the corresponding optical quantities can be expressed through the layers' gyrotropic characteristics. This approach is applied to calculate the specific rotation angle of plane of polarization of light propagating through a nonideal $1 D$-superlattice, which varies in composition as well as in layers' width.
\end{abstract}

Keywords: Light Propagation, Nonideal 1D-Superlattice, Specific Rotation Angle

\section{Introduction}

At present, there are numerous papers [1-4] dealing with the studies of the optical properties of perfect and imperfect dielectric superlattices. Urgency of the research is conditioned by electrical engineering and electronics needs in layered structures, as well as by the progress in the development of the theory of superlattice optical properties. The procedures used to calculate the transmission coefficients and refractive indices for light developed in [5-7] allow the frequency-concentration dependence is exposed and turn out to be useful in simulation of composite materials with preset parameters with no spatial dispersion taken into account. At the same time, the investigation of the gyrotropy of crystals is often the only way to determine stereo- and crystallochemical characteristics as well as the fine details in construction of respective space-dispersing structures. Such investigations are even more urgent as now there exists a large quantity of organic complexes and polymers, which are optically active due to structure peculiarities or to the optical activity of molecules they are composed of $[8,9]$.

The problem of finding polariton modes (necessary for calculating the gyrotropic characteristics) in space-dispersing superlattices has not been solved yet. At the same time, with thickness of layers composing a multilayer much larger than the characteristic scales of spatial dispersion, the specified quantities can be evaluated only approximately. In such a case, the contribution of indi- vidual layer to gyrotropy can be regarded independent. Thus, the corresponding optical quantities can be expressed through the layers gyrotropic characteristics. In this research, this approach is applied to calculate the specific angle of the light polarization plane rotation, the light being propagated in an imperfect 1D-superlattice with an arbitrary number of heterogeneous layers varying composition or thickness.

Here we consider light propagation along layer's optical axes perpendicular to their planes. An analytical expression is derived for the specific rotation angle as a function of the impurity layer concentration.

\section{Modeling}

According to the above-mentioned approach, in an imperfect topologically ordered one dimensional superlattice composed of $N$ unit cells, the angle of the light propagation plane rotation is described by the expression:

$$
\rho(\omega)=\sum_{n=1}^{N} \sum_{\alpha=1}^{\sigma} \rho_{n \alpha}(\omega) a_{n \alpha}
$$

We assume the quantity of $N$ to be larger enough (to neglect the effects from the influence of sample boundaries). In (1) $\rho_{n \alpha}(\omega)$ and $a_{n \alpha}(\omega)$ are the configuration-dependent specific angle of the light propagation plane rotation and thickness of the $\alpha$-th layer of the $n$-th unit cell; $\sigma$ is the number of unit-cell layers.

According to the general principles of the physics of 
disordered systems, the rotation angle measured experimentally should be equal to $\hat{P} \rho(\omega) \equiv\langle\rho(\omega)\rangle$, where $\hat{P}$ is the configuration averaging operator $[6,7,10]$ influencing the configuration-dependent function $\rho(\omega)$. In the imperfect $1 \mathrm{D}$-superlattice under consideration, the disordering is of two types, consequently, there are two types of configuration dependence. The first disorder is due to heterogeneous (defective) layers present in the superlattice, which differ from the perfect-system layers in physico-chemical composition (the configuration-dependent quantity is $\left.\rho_{n \alpha}(\omega)\right)$. The second is due to layersdefects present in the system and differing in thickness from the perfect superlattice (the configuration-dependent quantity is $\left.a_{n \alpha}\right)$. In what follows, we believe the disordering factors to be mutually independent. Quantities $\rho_{n \alpha}(\omega)$ and $a_{n \alpha}$ relate to configuration-dependent stochastic variables $\eta_{s \alpha}^{\mu(\alpha)}$ and $\eta_{s \alpha}^{v(\alpha)}$ as

$$
\rho_{n \alpha}(\omega)=\sum_{\mu(\alpha)=1}^{r(\alpha)} \rho_{\alpha}^{\mu(\alpha)} \eta_{n \alpha}^{\mu(\alpha)}
$$

and

$$
a_{n \alpha}=\sum_{v(\alpha)}^{s(\alpha)} a_{\alpha}^{v(\alpha)} \eta_{n \alpha}^{v(\alpha)}
$$

where

$$
\sum_{\mu(\alpha)=1}^{r(\alpha)} \eta_{n \alpha}^{\mu(\alpha)}=1, \sum_{\nu(\alpha)=1}^{s(\alpha)} \eta_{n \alpha}^{v(\alpha)}=1, \quad \eta_{n \alpha}^{\mu(\alpha)}=1, \text { if the } \alpha \text {-th layer }
$$
of the $n$-th unit cell is the layer of $\mu(\alpha)$-type $(\mu(\alpha)=1.2 \ldots r(\alpha))$ and $\eta_{s \alpha}^{\mu}=0$ - in any other case; $\eta_{n \alpha}^{v(\alpha)}=1$, if thickness of the $\alpha$-th layer of the $n$-th unit cell equals $a_{\alpha}^{v(\alpha)}(v(\alpha)=1.2 . . s(\alpha))$ and $\eta_{s \alpha}^{v}=0$ - in any other case. $\rho_{\alpha}^{\mu(\alpha)}(\omega)$ is the specific rotation angle of the $\alpha$-th layer of $\mu(\alpha)$ type. Now and then index $\mu$ enumerates layers of variable composition, $v$ - of variable thickness.

By using (1)-(3) as well as the averaging rules [10] for $\langle\rho(\omega)\rangle$, we have

$$
\begin{aligned}
\langle\rho(\omega)\rangle= & N \sum_{\alpha=1}^{\sigma}\left[\rho_{\alpha}^{(1)}(\omega) a_{\alpha}^{(1)}+a_{\alpha}^{(1)} \sum_{\mu(\alpha)=1}^{r(\alpha)} \Delta \rho_{\alpha}^{\mu(\alpha)}(\omega) C_{\alpha}^{\mu(\alpha)}\right. \\
& +\rho_{\alpha}^{(1)}(\omega) \sum_{v(\alpha)=1}^{s(\alpha)} \Delta a_{\alpha}^{v(\alpha)} C_{\alpha}^{v(\alpha)} \\
& \left.+\sum_{\mu(\alpha)=1}^{r(\alpha)} \sum_{v(\varepsilon)}^{s(\alpha)} \Delta \rho_{\alpha}^{\mu(\alpha)}(\omega) \Delta a_{\alpha}^{v(\alpha)} C_{\alpha}^{\mu(\alpha)} C_{\alpha}^{v(\alpha)}\right]
\end{aligned}
$$

$\Delta \rho_{\alpha}^{\mu(\alpha)}=\rho_{\alpha}^{\mu(\alpha)}-\rho_{\alpha}^{(1)}, \Delta a_{\alpha}^{v(\alpha)}=a_{\alpha}^{v(\alpha)}-a_{\alpha}^{(1)}$ as well as $C_{\alpha}^{\mu(\alpha)}, C_{\alpha}^{v(\alpha)}$ are concentrations of defect layers which differ from layers of base substance in composition and/or thickness, respectively. In expression (4), the fist summand corresponds to the rotation angle for the light polarization plane of perfect 1D-superlattice composed of layers of the (1)type (this substance is assumed to be basic). The second summand stands for superlattice compositional disorder, it goes to zero in the absence of composition variation. The third summand corresponds to disordering in thickness (with no disordering, the summand goes to zero). And the last summand stands for superlattice disordering in layer composition and thickness at a time. In the absence of any disordering, the fourth summand (4) goes to zero. In expression (4) each summand has meaning of rotation angle per unit cell. The angles, in contrast to $\rho_{n \alpha}^{\mu(\alpha), v(\alpha)}$ (measured in deg/unit length) are measured in degrees.

\section{Results}

For a more concrete results, let us consider the propagation of electromagnetic radiation in an imperfect aluminium organic 1D-superlattice with two elements-layers in a cell: the first is the aluminium menthylate $\mathrm{Al}(\mathrm{O}-\mathrm{Ment})_{3}$ layer $\left(\rho_{1}^{(1)}=-61,2\right)$, the second-aluminium bornylate $\mathrm{Al}(\mathrm{O} \text {-Born })_{3}$ layer $\left(\rho_{2}^{(1)}=-36,7\right)$. Let the first sublattice contain impurity layer l-MentOH $\left(\rho_{1}^{(2)}=-45,5\right)$, the second-l-BornOH $\left(\rho_{2}^{(2)}=-35,4\right)$ ones. Here we use values of specific rotation angles from [9]. In first and second sublattices, the concentration and the thickness of base-substance layer are denoted by $C_{1}^{(1)}, a_{1}^{(1)}$ and $C_{2}^{(1)}, a_{2}^{(1)}$, respectively, $C_{C(T) 1}^{(2)}, a_{1}^{(2)}$ and $C_{C(T) 2}^{(2)}, a_{2}^{(2)}$ are those of the impurity (index $C(T)$ stands for variation of impurity layers in composition or thickness). With formula (4) and by simple transformations we obtain the following concentration dependence of the specific angle of rotation

$$
\rho=\rho\left(C_{C(T) 1}^{(2)}, C_{C(T) 2}^{(2)}\right) \equiv\langle\rho\rangle / N\langle d\rangle
$$

(here $\langle d\rangle=a_{1}^{(1)}+a_{2}^{(1)}+\left(a_{1}^{(2)}-a_{1}^{(1)}\right) C_{T 1}^{(2)}+\left(a_{2}^{(2)}-a_{2}^{(1)}\right) C_{T 2}^{(2)}$ is the averaged period of the cell in 1D-sublattice) for the light polarization plane in two-sublattice imperfect 1Dsublattice in the form:

$$
\begin{aligned}
\rho= & \left(1+a+f_{1} C_{T 1}^{(2)}+f_{2} a C_{T 2}^{(2)}\right)^{-1}\left[\rho_{1}^{(1)}+a \rho_{2}^{(1)}\right. \\
& +\rho_{1}^{(1)} f_{1} C_{T 1}^{(2)}+\rho_{2}^{(1)} f_{2} a C_{T 2}^{(2)} \\
& +\left(\rho_{1}^{(2)}-\rho_{1}^{(1)}\right) \cdot\left(1+f_{1} C_{T 1}^{(2)}\right) C_{C 1}^{(2)} \\
& \left.+\left(\rho_{2}^{(2)}-\rho_{2}^{(1)}\right)\left(1+f_{2} a C_{T 2}^{(2)}\right) C_{C 2}^{(2)}\right]
\end{aligned}
$$


In (6), there is the following designation:

$a=a_{2}^{(1)} / a_{1}^{(1)}, f_{1}=\left(a_{1}^{(2)} / a_{1}^{(1)}\right)-1, f_{2}=\left(a_{2}^{(2)} / a_{2}^{(1)}\right)-1$.

The concentration dependence $\rho=\rho\left(C_{C(T) 1}^{(2)}, C_{C(T) 2}^{(2)}\right)$ of the specific angle of the light polarization plane rotation in the studied imperfect superlattice is graphically shown in Figures 1 and 2.

Figure 1 illustrates the presence of impurity layers in the first sublattice, which differ in composition, those in the second sublattice differ in thickness. In Figure 2 there is a variation of superlattice layers in thickness, Figure 3 shows the case when the layers vary in composition.

\section{Conclusions}

The paper is devoted to numerical simulation of the concentration dependence of the rotation angle for the light polarization plane in imperfect 1D-multilayer with an arbitrary number of sublattices. The choice of aluminium

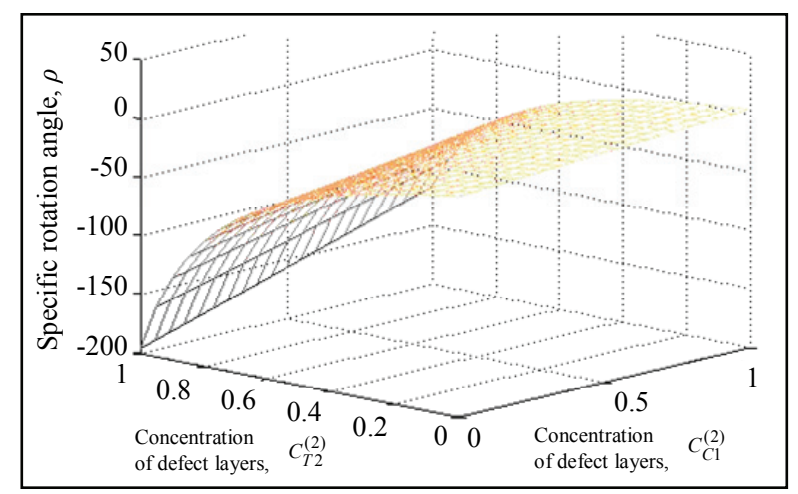

Figure 1. Concentration dependence $\rho=\rho\left(C_{C 1}^{(2)}, C_{T 2}^{(2)}\right)$, where $a_{1}^{(2)} / a_{1}^{(1)}=1, a_{1}^{(2)} / a_{1}^{(1)}=0.2, a_{2}^{(2)} / a_{2}^{(1)}=2$

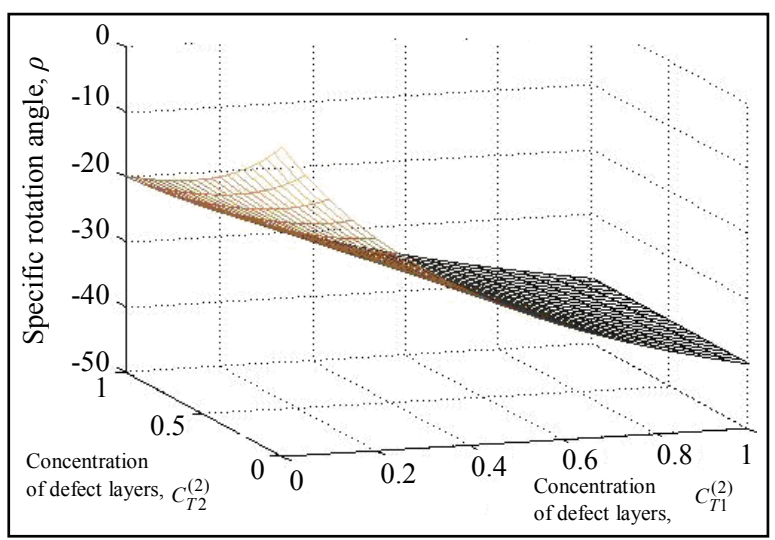

Figure 2. Concentration dependence $\rho=\rho\left(C_{T 1}^{(2)}, C_{T 2}^{(2)}\right)$ of the specific angle of the light polarization plane rotation in studied imperfect superlattice; $a_{1}^{(2)} / a_{1}^{(1)}=5$

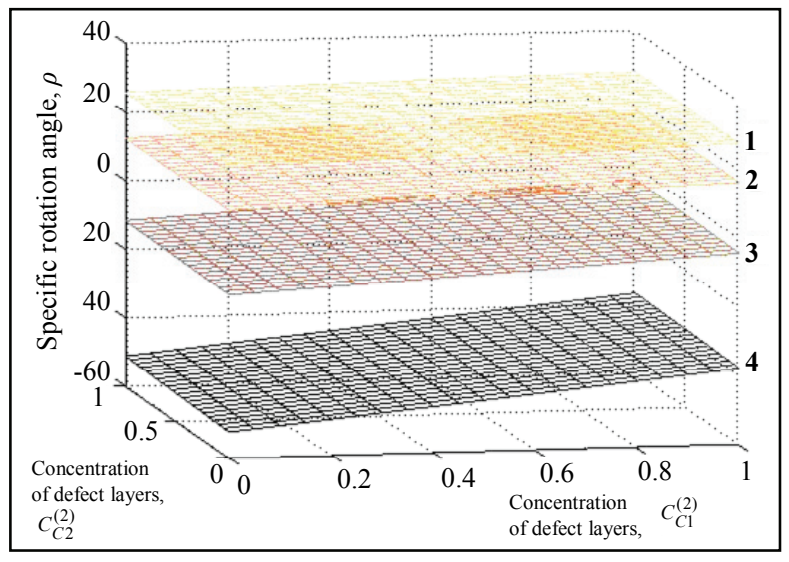

Figure 3. Concentration dependence $\rho=\rho\left(C_{C 1}^{(2)}, C_{C 2}^{(2)}\right)$ of the specific angle of the light polarization plane rotation in studied imperfect superlattice; $a_{1}^{(2)} / a_{1}^{(1)}=8, a_{2}^{(2)} / a_{2}^{(1)}=3$, $a_{2}^{(1)} / a_{1}^{(1)}$ being equal $8,3,1$ and 0.1 for case $1,2,3$ and 4 respectively

terpene alcogolates as objects of investigation is due to the wide application of these optically active materials in the capacity of catalysts of different asymmetric syntheses stimulating catalytic reactions of other optically active compounds [9].

At present, there exist a great number of organic complexes and polymers, which are optically active due to peculiarities of structure or optical activity of constituent molecules [8,9]. Studies of polymeric composite materials $[1,2,9]$ are urgent enough due to variety of properties and a wide range of application. A high interest in materials of this class has arisen, on the one hand, from demands of electrical engineering and electronics for thin films and layered structures and, on the other hand, it relates to the latest achievements in nanotechnologies and photonics [11], as well as a substantial progress in the theory of optical properties of perfect and imperfect layered structures.

\section{Acknowledgment}

The authors are grateful to Prof. Yurii Pashkevich for valuable discussions. This work is supported by Project "Dynamical and static properties of complex low-dimensional systems in external fields" of National Academy of Sciences of Ukraine.

\section{REFERENCES}

[1] C. Zhang and D. E. Hirt, "Layer-By-Layer Self-Assembly of Polyelectrolyte Multilayers on Cross-Section Surfaces of Multilayer Polymer Films: A Step toward Nano-Patterning Flexible Substrates," Polymer, Vol. 48, 2007, pp. 6748-6754.

[2] A. Pucci, M. Bernabò and P. Elvati, "Photoinduced For- 
mation of Gold Nanoparticles into Vinyl Alcohol Based Polymers," Journal of Materials Chemistry, Vol. 16, 2006, pp. 1058-1066.

[3] A. Kondilis and P. Tzanetakis, "Numerical Calculations on Optical Localization in Multilayer Structures with Random-Thickness Layers," Physical Review, Vol. B46, 1992, pp. 15426-15431.

[4] L. Lyubchanskii, N. N. Dadoenkova, M. L. Lyubchanskii, E. A. Shapovalov, A. Lakhtakia and Th. Rasing, "OneDimensional Bigyrotropic Magnetic Photonic Crystals," Applied Physics Letters, Vol. 85, 2004, pp. 5932-5934.

[5] A. Yariv and P. Yeh "Optical Waves in Crystals," John Willey \& Sons, New York, 1987.

[6] V. V. Rumyantsev, S. A. Fedorov and E. Ya. Shtaerman, "Peculiarities of Photonic Band Gap Width Dependence upon Concentration of the Admixture Layers Randomly Included in Composite Material," Functional Materials, Vol. 15, 2008, pp. 223-227.
[7] V. V. Rumyantsev and S. A. Fedorov, "Propagation of Light in a Quasi-Two-Dimensional $\mathrm{Si} / \mathrm{Sio}_{2}$ Superlattice with Variable Layer Thickness," Optics and Spectroscopy, Vol. 106, 2009, pp. 627-631.

[8] J. Ryan and R. A. L. Jones, "Polymers: The Quest for Motility," Materials Today, Vol. 11, 2008, pp. 21-23.

[9] M. L. Sierra, R. Kumar, V. S. J. de Mel and J. P. Oliver, "Synthesis and Spectroscopic Investigations of Alkylaluminum Alkoxides Derived from Optically Active Alcohols. The First Structural Identification of an Optically Active Organoaluminum Alkoxide," Organometallics, Vol. 11, 1992, pp. 206-214.

[10] M. Ziman, "Models of Disorder," John Willey \& Sons, New York, 1979

[11] D. Joannopoulos, S. G. Johnson, J. N. Winn and R. D. Meade, Photonic Crystals, 2nd Edition, Princeton University Press, Princeton, 2008. 